\title{
Results of The SIGNET Trial - a randomised controlled trial of glutamine and/or selenium supplemented parenteral nutrition in critical illness
}

\author{
P. J. Andrews ${ }^{1}$, A. Avenell ${ }^{2}$ and The Signet Trial Group \\ ${ }^{1}$ Department of Anaesthesia, Critical Care and Pain Medicine, University of Edinburgh, Western General Hospital, \\ Edinburgh EH4 2XU, UK and ${ }^{2}$ Health Services Research Unit, University of Aberdeen, AB25 2ZD, UK
}

The SIGNET trial was a randomised, factorial design, controlled trial of parenteral glutamine (20.2 g/d) and/or selenium supplementation $(500 \mu \mathrm{g} / \mathrm{d})$ in adult patients in intensive care unit (ICUs) and high dependency units (HDUs) for at least $48 \mathrm{~h}$, requiring parenteral nutrition (PN). Supplementation was for up to seven days, unless death occurred or PN was discontinued.

The primary outcomes were whether each participant had an infection (all infections, and Centres for Disease Control confirmed infections) within the first 14 days, and all cause mortality. Secondary outcomes were ICU, HDU and acute hospital length of stay; days of antibiotic use; sepsis-related organ failure assessment score; serious adverse events; quality of life assessed by SF12 and EQ5D.

Enrolment of 502 participants from 10 hospitals in Scotland was completed in December 2008. Participants in the four groups were well balanced at trial entry. The majority of patients were surgical and the mean age was 64 years. More than half of the patients had sepsis at recruitment, and just over a quarter were under-nourished.

\begin{tabular}{|c|c|c|c|c|}
\hline & Glutamine & Selenium & Glutamine and selenium & Neither \\
\hline Randomised $N$ & 126 & 127 & 124 & 125 \\
\hline Age mean (SD) & $63.2(14.5)$ & $64.5(14.2)$ & $64.5(15.7)$ & $63.1(15.3)$ \\
\hline Sex $(\%$ female $)$ & 38.9 & 39.4 & 39.5 & 40.0 \\
\hline Medical patients $(\%)$ & 23.8 & 23.6 & 29.0 & 24.8 \\
\hline APACHE II score mean (SD) & $21.2(7.4)$ & $20.8(7.3)$ & $20.1(5.8) \S$ & $20.0(6.9) \#$ \\
\hline Sepsis $(\%)$ & $61.6^{*}$ & $53.6^{*}$ & 58.9 & $52.4 \#$ \\
\hline Undernourished $(\%)$ & 27.8 & 28.3 & 25.8 & 27.2 \\
\hline Days of SIGNET parenteral nutrition mean (SD) & $4.62(2.22)$ & $4.30(2.30)$ & $4.72(2.21)$ & $4.40(2.10)$ \\
\hline
\end{tabular}

In an intention to treat analysis there was no statistically significant reduction in participants with infections from glutamine (odds ratio, OR, $1.07,95 \%$ CI $0.75-1.53$ ) or selenium (OR $0.81,95 \%$ CI $0.57-1.15$ ). In the prespecified subgroup analysis of those people who received at least 5 days of supplementation or died before this time, the corresponding figures were glutamine OR 1.08 (95\% CI $0.66-$ 1.76) and selenium OR 0.61 (95\% CI 0.37-0.99). There was no significant effect on mortality or lengths of stay.

We thank the Medical Research Council, Chief Scientist Office of the Scottish Government Health Directorates, Fresenius Kabi and Oxford Nutrition for funding. We thank The Scottish Intensive Care Society, and participants and their relatives for support. We thank all the staff in the ICUs and HDUs for their collaboration and support, which was essential for the conduct of the trial. 